\title{
Asymptotic Behavior of a Generalized Polynomial
}

\author{
Anwar Habib
}

Department of General Studies Jubail Industrial College Al Jubail , K S A

Abstract: We have extended the corresponding result of Voronowskaja for Lebesgue integrable function in $L_{1}$ norm by our newly defined Generalized Polynomial.

where $\quad p_{n, k}(x ; \alpha)=\left(\begin{array}{l}n \\ k\end{array}\right) \frac{x(x+k \alpha)^{k-1}(1-x+(n-k) \alpha)^{n-k}}{(1+n \alpha)^{n}}$

$$
A_{n}^{\alpha}(f, x)=(n+1) \sum_{k=0}^{n}\left\{\int_{k /(n+1)}^{(k+1) /(n+1)} f(t) d t\right\} p_{n, k}(x ; \alpha)
$$

Keywords: Bernstein Polynomials, Convergence, Generalized Polynomials, Integrable function, $L_{1}$ norm,

\section{Introduction and Results}

If $f(x)$ is a function defined [0,1], the Bernstein polynomial $B_{n}^{f}(x)$ of $f$ is given as

$B_{n}^{f}(x)=\sum_{k=0}^{n} f(k / n) p_{n, k}(x)$

where

$p_{n, k}(x)=\left(\begin{array}{l}n \\ k\end{array}\right) x^{k}(1-x)^{n-k}$

One question arises about the rapidity of convergence of $B_{n}^{f}(\mathrm{x})$ to $f(x)$. An answer to this question has been given in different directons. One direction is that in which $f(x)$ is supposed to be at least twice differentiable in a point $x$ of $[0,1]$.

Voronowskaja [6] proved that

$\lim _{n \rightarrow \infty} n\left|f(x)-B_{n}^{f}(x)\right|=-\frac{1}{2} x(1-x) f^{\prime \prime}(x)$.

In particular, if $f^{\prime \prime}(x) \neq 0$, difference $f(x)-B_{n}^{f}(x)$ is exactly of order $n^{-1}$

A small modification of Bernstein polynomial due to Kantorovitch [4] makes it possible to approximate Lebesgue integrable function in $L_{1}$-norm by the modified polynomials

${ }_{n}^{f}(x)=(n+1) \sum_{k=0}^{n}\left\{\int_{k /(n+1)}^{(k+1) /(n+1)} f(t) d t\right\} p_{n, k}(x) \quad \ldots(1.4)$

where $p_{n, k}(x)$ is defined by (1.2)

By Abel's formula(see Jensen [3])

$(x+y+n \alpha)^{n}=\sum_{k=0}^{n}\left(\begin{array}{l}n \\ k\end{array}\right) x(x+k \alpha)^{k-1}(y+(n-k) \alpha)^{n-k}$

If we put $=1-x$, we obtain (see Cheney and Sharma [2] )

$(1+n \alpha)^{n}=\sum_{k=0}^{n}\left(\begin{array}{l}n \\ k\end{array}\right) x(x+k \alpha)^{k-1}(1-x+(n-k) \alpha)^{n-k}$

Or

$1=\sum_{k=0}^{n}\left(\begin{array}{l}n \\ k\end{array}\right) \frac{x(x+k \alpha)^{k-1}(1-x+(n-k) \alpha)^{n-k}}{(1+n \alpha)^{n}}$

Thus defining

$p_{n, k}(x ; \alpha)=\left(\begin{array}{l}n \\ k\end{array}\right) \frac{x(x+k \alpha)^{k-1}(1-x+(n-k) \alpha)^{n-k}}{(1+n \alpha)^{n}}$

we have

$\sum_{k=0}^{n} p_{n, k}(x ; \alpha)=1$

and (see Anwar Habib [1])

$$
\sum_{\mathrm{k}=0}^{\mathrm{n}} \mathrm{kp}_{\mathrm{n}, \mathrm{k}}(\mathrm{x} ; \alpha) \leq \mathrm{nx} /(1+\alpha)-\text {---------------1.9 }
$$

$\left.\sum_{k=0}^{n} k^{2} p_{n, k}(x ; \alpha) \leq\right) \frac{n x(1-x)+n^{2} x(x+\alpha)}{1+\alpha}$ $-1.10$ 
we now define the Generalized Polynomial

$$
A_{n}^{\alpha}(f, x)=(n+1) \sum_{k=0}^{n}\left\{\int_{k /(n+1)}^{(k+1) /(n+1)} f(t) d t\right\} p_{n, k}(x ; \alpha)
$$

where $p_{n, k}(x ; \alpha)$ is defined in (1.7) and moreover when $\alpha=0,(1.7)$ and (1.11) reduces to (1.2) and (1.4) respectively.

In this paper, we shall prove the corresponding results of approximation due to Voronowskja[6] for Lebesgue integrable function in $L_{1}$-norm by the our polynomial (1.11). In fact we state our result is as follows of Theorem: let $f(x)$ be bounded Lebesgue integrable function with its first derivative in [0,1] and suppose that the second derivative $f^{\prime \prime}(x)$ exists at a certain point $x$ of $[0,1]$,

then for $\alpha=\alpha_{n}=o(1 / n)$

$$
\lim _{n \rightarrow \infty} n\left[A_{n}^{\alpha}(f, x)-f(x)\right]=\frac{1}{2}\left[(1-2 x) f^{\prime}(x)-x(1-x) f^{\prime \prime}(x)\right]
$$

\section{Lemma}

we first like to prove the lemma which would be useful for the proof of our theorem

Lemma - For all values of $x \in|0,1|$ and for $\alpha=\alpha_{n}=o(1 / n)$, we have

Proof of Lemma:

$$
(n+1) \sum_{k=0}^{n}\left\{\int_{k /(n+1)}^{(k+1) /(n+1)}(t-x)^{2} d t\right\} p_{n, k}(x ; \alpha) \leq x(1-x) / n
$$

$$
\begin{aligned}
&(n+1) \sum_{k=0}^{n}\left\{\int_{k /(n+1)}^{(k+1) /(n+1)}(t-x)^{2} d t\right\} p_{n, k}(x ; \alpha) \\
&= \sum_{k=0}^{n}\left[x^{2}-\frac{2 k x+x}{n+1}+\frac{k^{2}+k}{(n+1)^{2}}+\frac{1}{3(n+1)^{2}}\right] p_{n, k}(x ; \alpha) \\
& \leq x^{2}-\frac{1}{(n+1)}\left[\frac{2 n x^{2}}{1+\alpha}+x\right]+\frac{1}{(n+1)^{2}}\left[n(n-1) x \frac{x+2 \alpha}{(1+2 \alpha)^{2}}\right. \\
&+\left.\frac{(n-2) \alpha^{2}}{(1+3 \alpha)^{3}}+\frac{2 n x}{1+\alpha}\right]+\frac{1}{3(n+1)^{2}} \\
& \leq \frac{1}{n(1-\alpha)(1+2 \alpha)^{2}(1+3 \alpha)^{3}}[x(1-x)+\alpha x(1-x)(2 n+9)+x \\
& \quad+\alpha^{2} x(1-x)(17 n+23)+9 x \\
& \quad+\alpha^{3} x(1-x)(57 n-13)+7 n x^{2}+x\left(5 n^{2}+35\right) \\
& \quad+\alpha^{4} x(1-x)(96 n-144)+86 n x^{2}+x\left(65 n^{2}+12 n\right) \\
& \quad+\alpha^{5} x(1-x)(54 n-216)+x(4 n-12 n+46)+162 n x^{2} \\
&\left.\quad+\alpha^{6} 108 x(1-x)+108 n x^{2}\right]+1 / 3 n^{2} \\
& \leq \frac{x(1-x)}{n} \text { for } \alpha=\alpha \mathrm{n}=\mathrm{o}\left(\frac{1}{n}\right) \text { and for large } \mathrm{n} \\
&
\end{aligned}
$$

which completes the proof of Lemma.

\section{Proof of Theorem}

\section{Proof of the Theorem}

We write(in view of Taylor's Theorem)

$f(t)=f(x)+(t-x) f^{\prime}(x)+(t-x)^{2}\left[\frac{1}{2} f^{\prime \prime}(x)+\eta(t-x)\right]$

where $\eta(h)$ is bounded $|\eta(h)| \leq H$ for all $\mathrm{h}$ and converges to zero with $\mathrm{h}$.

Multiplying eqn. $(2.1)$ by $(n+1) p_{n, k}(x ; \alpha)$ and integrating it from $k /(n+1)$ to $(k+1) /(n+1)$, then on summing ,we get

$$
(n+1) \sum_{k=0}^{n}\left\{\int_{k /(n+1)}^{(k+1) /(n+1)} f(t) d t\right\} p_{n, k}(x ; \alpha)
$$




$$
\begin{gathered}
=(n+1) \sum_{k=0}^{n}\left\{\int_{k /(n+1)}^{(k+1) /(n+1)} f(x) d t\right\} p_{n, k}(x ; \alpha) \\
+(n+1) \sum_{k=0}^{n}\left\{\int_{k /(n+1)}^{(k+1) /(n+1)} f(t-x) f^{\prime}(x) d t\right\} p_{n, k}(x ; \alpha) \\
+\frac{1}{2}(n+1) \sum_{k=0}^{n}\left\{\int_{k /(n+1)}^{(k+1) /(n+1)}(t-x)^{2} f^{\prime \prime}(x) d t\right\} p_{n, k}(x ; \alpha) \\
+(n+1) \sum_{k=0}^{n}\left\{\int_{k /(n+1)}^{(k+1) /(n+1)}(t-x)^{2} \eta(t-x) d t\right\} p_{n, k}(x ; \alpha) \\
=I_{1}+I_{2}+I_{3}+I_{4} \text { (say) }
\end{gathered}
$$

Now first we evaluate $I_{1}$ :

$=f(x)$

$$
I_{1}=(n+1) \sum_{k=0}^{n}\left\{\int_{k /(n+1)}^{(k+1) /(n+1)} f(x) d t\right\} p_{n, k}(x ; \alpha)
$$

and then

$$
\begin{gathered}
I_{2}=(n+1) \sum_{k=0}^{n}\left\{\int_{k /(n+1)}^{(k+1) /(n+1)} f(t-x) f^{\prime}(x) d t\right\} p_{n, k}(x ; \alpha) \\
=\sum_{k=0}^{n}\left(\frac{2 k+1}{2(n+1)}-x\right) f^{\prime}(x) p_{n, k}(x ; \alpha)
\end{gathered}
$$

$\leq \frac{(1-2 x)}{2 n} f^{\prime}(x)$ for $\alpha=\alpha_{n}=o(1 / n)$

Now we evaluate $I_{3}$ :

$$
\begin{aligned}
& I_{3}=\frac{1}{2}(n+1) \sum_{k=0}^{n}\left\{\int_{k /(n+1)}^{(k+1) /(n+1)}(t-x)^{2} f^{\prime \prime}(x) d t\right\} p_{n, k}(x ; \alpha) \\
& \leq x(1-x) f^{\prime \prime}(x) / 2 n \text { (by lemma) }
\end{aligned}
$$

and then in the last we evaluate $I_{4}$ :

$$
I_{4}=(n+1) \sum_{k=0}^{n}\left\{\int_{k /(n+1)}^{(k+1) /(n+1)}(t-x)^{2} \eta(t-x) d t\right\} p_{n, k}(x ; \alpha)
$$

$I_{4}$ can be estimated easily. Let $\epsilon>0$ be arbitrary $\delta>0$ such that $|\eta(h)|<\epsilon$ for $|h|<\delta$ thus breaking up the sum $I_{9}$ into two parts corresponding to those values of $t$ for which $|\mathrm{t}-x|<\delta$, and since in the given range of $\mathrm{t},\left|\frac{k}{n}-x\right| \sim|t-x|$, we have

$$
\begin{aligned}
\left|I_{4}\right| \leq \epsilon & \sum_{\left|\left(\frac{k}{n}\right)-x\right|<\delta}(n+1) p_{n, k}(x ; \alpha)\left|\int_{\frac{k}{n+1}}^{\frac{k+1}{n+1}}(t-x)^{2} d t\right| \\
& +H \sum_{\left|\left(\frac{k}{n}\right)-x\right| \geq \delta}^{\frac{k+1}{n+1}}(n+1) p_{n, k}(x ; \alpha)\left|\int_{\frac{k}{n+1}} d t\right|
\end{aligned}
$$

$=I_{5}+I_{6}$ (say)

$\left|I_{5}\right| \leq \frac{\epsilon}{n}|\{x(1-x)\}|$, for $\alpha=\alpha_{n}=o(1 / n)$ 


$$
\begin{aligned}
I_{6}= & (n+1) H \sum_{\left|\left(\frac{k}{n}\right)-x\right| \geq \delta}\left\{\int_{\frac{k}{n+1}}^{\frac{k+1}{n+1}} d t\right\} p_{n, k}(x ; \alpha) \\
= & (n+1) \sum_{\left|\left(\frac{k}{n}\right)-x\right| \geq \delta} p_{n, k}(x ; \alpha) \frac{1}{n+1}
\end{aligned}
$$

But if $=n^{-\beta}, 0<\beta<1 / 2$ (see also Kantorovitch [4]),

then for $\alpha=\alpha_{n}=o(1 / n)$

For $\mathrm{v}>0$, the constant $\mathrm{C}=\mathrm{C}(\beta, \mathrm{v})$.

$$
\sum_{\left|\left(\frac{k}{n}\right)-x\right| \geq n-\beta} p_{n, k}(x ; \alpha) \leq C n^{-v}
$$

whence $I_{6}<\frac{\epsilon}{n+1}<\epsilon / n$ for all $n$ sufficiently large and therefore it follows

$I_{4}<\epsilon / n$, for all sufficiently large $\mathrm{n}$

Hence from (2.2), (2.3), (2.4), (2.5) and (2.6), we have

$$
\begin{gathered}
(n+1) \sum_{k=0}^{n}\left\{\int_{k /(n+1)}^{(k+1) /(n+1)} f(t) d t\right\} p_{n, k}(x ; \alpha) \\
=f(x)+\left[\left\{(1-2 x) f^{\prime}(x)+x(1-x) f^{\prime \prime}(x)\right\} / 2 n\right]+(\epsilon / n)
\end{gathered}
$$

and therefore, finally we get

where $\epsilon \rightarrow 0$ as $n \rightarrow \infty$

$$
\lim _{n \rightarrow \infty} n\left[A_{n}^{\alpha}(f, x)-f(x)\right]=\frac{1}{2}\left[(1-2 x) f^{\prime}(x)-x(1-x) f^{\prime \prime}(x)\right]
$$

which completes the proof of the theorem.

\section{Conclusion}

In this paper we have extended the result of Voronowskaja by taking a Generalized Polynomials $A_{n}^{f}(x)$ instead of Bernstein Polynomial ${ }_{n}^{f}(x)$

\section{References}

[1] Anwar Habib (1981). On the degree of approximation of functions by certain new Bernstein type Polynomials. Indian J. pure Math. ,12(7):882-888.

[2] Cheney, E.W. , and Sharma, A.(1964). On a generalization of Bernstein polynomials.Rev. Mat. Univ. Parma(2),5,77-84.

[3] Jensen, J. L. W. A. (1902). Sur une identité Abel et sur d'autress formules amalogues. Acta Math. , 26, 307-18

[4] Kantorovitch, L.V.(1930). Sur certains développments suivant lés pôlynômes dé la forme S. Bernstein I,II. C.R. Acad. Sci. URSS, 20,563-68,595-600.

[5] Lorentz, G.G. (1955). Bernstein Polynomials. University of Toronto Press, Toronto

[6] Voronowskaja, E. (1932). Determination de la forme asymtotique d' d noitamixorppaé 1 noitcnof sép sônylô M ed sem Bernstein. C.R. Acad. Sci. URSS,22,79-85 